See Article page 100.

\section{Commentary: Simplifying the simple}

\author{
Jonathan C. Nesbitt, MD
}

The ancient phrase practice makes perfect overstates the simple fact that practice at most things produces skill improvement, not necessarily perfection. The goal is proficiency.

In cardiac surgery training, as in many instructional domains, specific skills are frequently and best taught by breaking down procedures and techniques into component parts, to make tasks simple and understandable. Simplifying the simple in our complex world of thoracic surgery requires a creative mind, ingenuity, experience, and perhaps a few toys. Albert Einstein noted that "everything should be made as simple as possible, but no simpler." Such a statement emphasizes the point that we need not focus on such detail as to obfuscate the global message. First concentrate on the basics and introduce the complexities accordingly.

Fundamental skills in performing cardiac surgery are optimally taught and applied through simulation, a proven and established methodology. This controlled environment for instructional practice provides the best opportunity for competence and proficiency before the real event. Resident physicians in cardiac surgery, particularly junior residents, need such time and instruction to establish aptitude in foundational tasks. As skills are established, more intricate training is introduced in addition to time and experience in operating rooms.

Kelly and colleagues ${ }^{1}$ present the results of a survey of cardiothoracic resident physicians on attitudes regarding the utility and importance of practice of technical skills.

From the Department of Thoracic Surgery, Vanderbilt University Medical Center, Nashville, Tenn.

Disclosures: The author reported no conflicts of interest.

The Journal policy requires editors and reviewers to disclose conflicts of interest and to decline handling or reviewing manuscripts for which they may have a conflict of interest. The editors and reviewers of this article have no conflicts of interest

Received for publication May 31, 2021; revisions received May 31, 2021; accepted for publication June 5, 2021; available ahead of print June 10, 2021.

Address for reprints: Jonathan C. Nesbitt, MD, Department of Thoracic Surgery, Vanderbilt University Medical Center, 609 Oxford House, 131321 st Ave S, Nashville, TN 37232 (E-mail: jon.nesbitt@vumc.org).

JTCVS Techniques 2021;8:114-5

2666-2507

Copyright (C) 2021 The Author(s). Published by Elsevier Inc. on behalf of The American Association for Thoracic Surgery. This is an open access article under the CC BY-NC-ND license (http://creativecommons.org/licenses/by-nc-nd/4.0/).

https://doi.org/10.1016/j.xjtc.2021.06.010

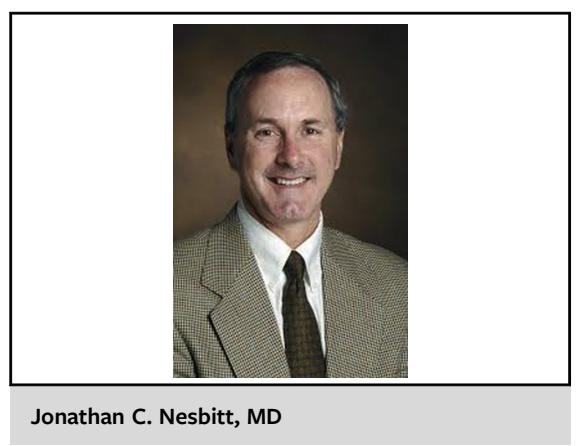

CENTRAL MESSAGE

The authors present results of a survey of cardiothoracic surgery resident physicians regarding the utility of practice of technical skills and propose low-cost simulators as a resource for training.

Survey respondents overwhelmingly emphasized the importance of practice. The authors recognize known impediments to such endeavors (eg, time commitment, faculty instruction, and motivation) and propose simple, portable, low-cost, and reusable simulators that they developed to facilitate and to use as a resource for training basic cardiac surgery skills (eg, suturing and cannulation). Their models are versatile and allow ad hoc practice in any environment. Anecdotally, the authors point out that resident physicians in their program had a smoother entry into operating rooms after practicing with their low fidelity simulators.

Simple task trainers in cardiac surgery have been available for many years. Annually, the Thoracic Surgery Directors Association provides pocket anastomosis trainers to all programs, and a well-vetted cardiac simulation curriculum exists on the Thoracic Surgery Directors Association website. The models proposed by Kelly and colleagues ${ }^{1}$ are additional alternatives at a low cost, with seemingly ease of use along with the ability to practice cannulation skills. Despite a creative set of models, the authors have an unproven and unvalidated commodity; there are no data or outcomes analytics from its use. A follow-up analysis of the devices with details of their use is essential before general acceptance and endorsement of their endeavor.

I applaud the authors' initiative to improve colleague training and their ingenuity in prototype development. Yet whatever the model, practice with feedback remains the key to success. If creative, low-cost, low-fidelity simulators as proposed by the authors can affect surgical competence 
by facilitating access and use, such strategies should rule the day. The proof will remain with model testing, training, and ultimately real-time proficiency. It seems simple.

\section{Reference}

1. Kelly JJ, Han JJ, Patrick WL, Mays JC, Iyengar A, Helmers MR, et al Do-it-yourself simulators and building a culture of practice in the virtual era J Thorac Cardiovasc Surg Tech. 2021;8:100-11. 